\title{
Suppressing autophagy: a strategy by Escherichia coli 0157:H7 for its survival on host epithelial cells
}

\author{
Yansong Xue and Mei-Jun Zhu'
}

\section{Introduction}

The primary function of autophagy is to degrade aged or impaired organelles, thus allowing cells to survive under extreme environments and rejuvenate cellular function. Besides the self-fulfillment function, autophagy also serves as a defense mechanism to invasive bacteria. Upon infection, autophagy is activated to engulf vesicles containing pathogenic bacteria for degradation. Conversely, to persist in host cells, coevolved pathogens develop sophisticated tactics to evade the trap of autophagy using various strategies. A recent publication shows that RavZ, a cysteine protease-like factor of intracellular pathogen Legionella pneumophila decreases the microtubule-associated protein 1 light chain-3B II (LC3BII) level by irreversibly blocking the conversion from LC3BI to LC3BII ${ }^{1}$. Likewise, pathogenicity islands 2 (SPI-2) of Salmonella Typhimurium manipulates host autophagy by actively regulating focal adhesion kinase $(\mathrm{FAK})^{2}$.

Escherichia coli O157:H7 is a gram-negative extracellular pathogen that produces Shiga toxins causing hemorrhagic diarrhea and hemolytic-uremic syndrome. In addition, it contains type 3 secretion system (T3SS) apparatus and effectors such as translocated intimin receptor (Tir) and E. coli secreted proteins (Esps). These effectors lead to intimate adhesion to host intestinal epithelial cells and formation of attachment and effacement (A/E) lesions ${ }^{3}$.

Despite evidence that manipulation of the host autophagic process is critical in the pathogenesis of invasive pathogens, the interaction of autophagy with extracellular

\footnotetext{
Correspondence: M-J. Zhu (meijun.zhu@wsu.edu)

'School of Food Science, Washington State University, Pullman, WA 99164, USA

Edited by G. Melino
}

pathogen E. coli O157:H7 is sparsely studied. Recent publications show that Shiga toxin serves as an autophagy inducer in immune cells and intestinal epithelial cells, likely through the induction of endoplasmic reticulum (ER) stress $^{4,5}$; Shiga toxin 2 induces DNA-damageinducible transcript 3 (DDIT3) activation and Akt1 inactivation, further leading to autophagic cell death ${ }^{4}$. Our recent publication in Cell Death Discovery reported that E. coli O157:H7 subverted host autophagic response to promote epithelial adhesion, which was mediated by T3SS effector, Tir $^{6}$ (Fig. 1). E. coli O157:H7 infection inhibited autophagy activity in colonic epithelial cells, which was abolished by tir deletion and was further restored by complementary tir plasmid expression ${ }^{6}$.

Tir has multiple residues that can be modified by host protein kinases. Upon adhesion to the host cells, Tir recruits various host protein kinases that facilitate epithelial adhesion, potentially interfering with host autophagy. Besides tyrosine phosphorylation, Tir is subjected to serine phosphorylation by host kinases such as cAMPactivated protein kinase $\mathrm{A}(\mathrm{PKA})^{7}$. We observed a robust PKA activation in response to $E$. coli $\mathrm{O} 157: \mathrm{H} 7$ infection but not in tir deletion mutant $(\Delta t i r)$ strain infected cells, indicating Tir mediated PKA activation. We further showed that PKA activation was essential for E. coli O157: H7-induced autophagy inhibition, blocking PKA abolished $E$. coli O157:H7 ability to inhibit autophagy ${ }^{6}$. Correspondingly, cholera toxin and edema toxin from Vibrio cholerae and Bacillus anthracis, respectively, induce PKA hyperactivation and reduce autophagosome formation ${ }^{8}$. The activation of PKA specifically phosphorylates autophagic proteins Atg1 and Atg13 and impairs their localization to phagophore9. S. Typhimurium selectively activates non-receptor tyrosine kinase FAK, thereby retarding the recruitment of LC3B to the 


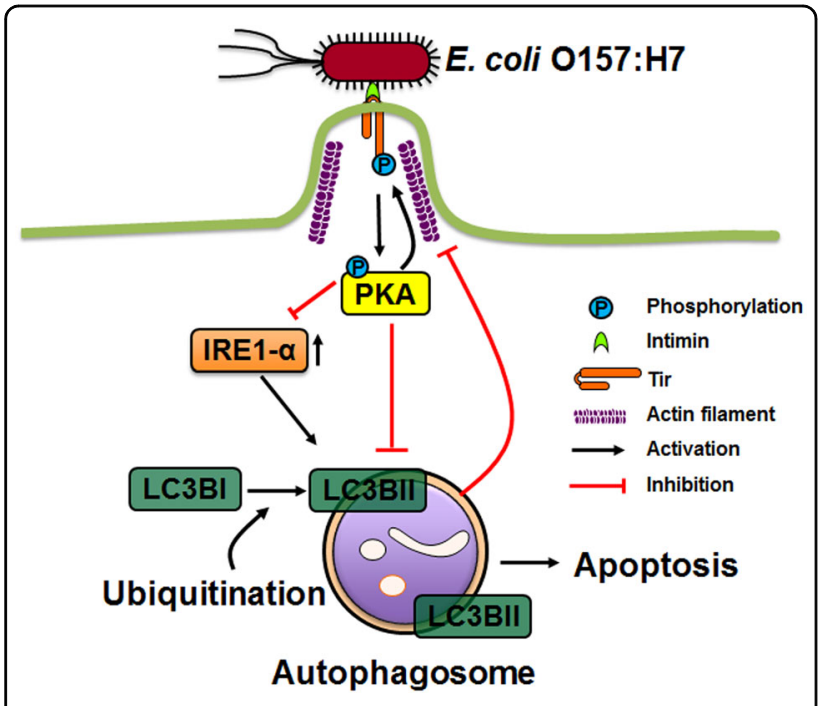

Fig. 1 Schematic diagram of autophagy inhibition by $E$. coli 0157:H7. Adhesion of E. coli O157:H7 activates PKA through translocated intimin receptor (Tir), which inhibits endoplasmic reticulum stress, and blocks autophagosome formation. Activation of autophagy disturbs the adhesion of $\mathrm{E}$. coli $\mathrm{O} 157: \mathrm{H7}$ to host cells with an undetermined mechanism.

autophagosomes ${ }^{2}$. The above data show that host protein kinases are hijacked by bacterial factors and play mediatory roles in autophagy regulation.

E. coli O157:H7 infection causes the disturbance of ER function ${ }^{4}$. ER stress is a potent inducer in autophagy activation resulting from accumulation of unfolded protein response $(\mathrm{UPR})^{10}$. The ER stress sensor inositolrequiring enzyme $1 \alpha$ (IRE1 $\alpha)$ is one of the mediators of UPR and has been implicated in ER stress-induced autophagy $^{11}$. In our recent publication, upon E. coli O157:H7 infection, IRE1- $\alpha$ was decreased ${ }^{6}$, which may reduce autophagy-induced cell death and benefit bacterial survival. Accumulating studies have indicated that autophagy-induced cell death is a host strategy to inhibit bacterial replication. Macrophage apoptosis caused by Salmonella infection is highly associated with autophagy hyperactivation induced by T3SS factor $\mathrm{SipB}^{12}$, which halts the replication of intracellular Salmonella effectively. Corresponding to inhibited autophagy, E. coli O157:H7 infection inhibited caspase- 8 and poly (ADP-ribose) polymerase (PARP) cleavage, suggesting that infection decelerated cell death process ${ }^{6}$.

Our recent publication in Cell Death Discovery further showed that host autophagy activation by starvation or rapamycin reduced the adherence of $E$. coli $\mathrm{O} 157: \mathrm{H} 7$ to HT-29 cells, while autophagy inhibition by chloroquine enhanced its epithelial adhesion ${ }^{6}$. It is consistent with our previous publication that tumor necrosis factor (TNF) reduced E. coli $\mathrm{O} 157: \mathrm{H} 7$ attachment to epithelial cells, which may be partially due to the activated autophagy ${ }^{13}$. Collectively, these data suggest that inhibition of host autophagy favors bacterial interaction with host cells; however, the detailed mechanism of how autophagy affects bacterial attachment warrants further research.

In summary, our recent paper in Cell Death Discovery demonstrates that $E$. coli $\mathrm{O} 157: \mathrm{H} 7$ has evolved strategies to block host autophagic response partially via activation of PKA that promotes its epithelial adhesion. Our findings provide new insight in the fight against $E$. coli $\mathrm{O} 157$ : $\mathrm{H} 7$ infection. The drugs/chemicals targeting PKA activation or boosting autophagy activity could be used to reduce/eliminate $E$. coli $\mathrm{O} 157: \mathrm{H} 7$ gut colonization and infection.

\section{Acknowledgements}

We thank Mr. Michael H. Taylor for his critical reading. This work was partially supported by USDA-AFRI 2010-65201-20599, Emerging Research Issues Competitive Grant and BioAg Grant from CAHNR at Washington State University.

\section{Competing interests}

The authors declare no conflict of interest.

\section{Publisher's note}

Springer Nature remains neutral with regard to jurisdictional claims in published maps and institutional affiliations.

Received: 19 October 2017 Accepted: 23 October 2017

Published online: 19 January 2018

\section{References}

1. Choy, A. et al. The legionella effector RavZ inhibits host autophagy through irreversible Atg8 deconjugation. Science 338, 1072-1076 (2012).

2. Owen, K. A., Meyer, C. B., Bouton, A. H. \& Casanova, J. E. Activation of focal adhesion kinase by Salmonella suppresses autophagy via an Akt/mTOR signaling pathway and promotes bacterial survival in macrophages. PLoS Pathog. 10, e1004159 (2014)

3. Stevens M. P., Frankel G. M. The locus of enterocyte effacement and associated virulence factors of enterohemorrhagic Escherichia coli. Microbiol. Spectr. 2 EHEC-0007-2013 (2014).

4. Tang, B. et al. Shiga toxins induce autophagic cell death in intestinal epithelial cells via the endoplasmic reticulum stress pathway. Autophagy 11, 344-354 (2015).

5. Lee, M. S. et al. Shiga toxins induce autophagy leading to differential signalling pathways in toxin-sensitive and toxin-resistant human cells. Cell. Microbiol. 13, 1479-1496 (2011).

6. Xue, Y., Du, M., Sheng, H., Hovde, C. J. \& Zhu, M. J. Escherichia coli O157: H7 suppresses host autophagy and promotes epithelial adhesion via Tirmediated and CAMP-independent activation of protein kinase A. Cell Death Discov. 3, 17055 (2017).

7. Allen-Vercoe, E., Waddell, B., Toh, M. C. \& DeVinney, R. Amino acid residues within enterohemorrhagic Escherichia coli O157:H7 Tir involved in phosphorylation, alpha-actinin recruitment, and Nck-independent pedestal formation. Infect. Immun. 74, 6196-6205 (2006).

8. Shahnazari, S., Namolovan, A., Mogridge, J., Kim, P. K. \& Brumell, J. H. Bacterial toxins can inhibit host cell autophagy through CAMP generation. Autophagy 7, 957-965 (2011).

9. Stephan, J. S., Yeh, Y. Y., Ramachandran, V., Deminoff, S. J. \& Herman, P. K. The Tor and PKA signaling pathways independently target the Atg1/Atg13 protein 
kinase complex to control autophagy. Proc. Natl Acad. Sci. USA 106, 17049-17054 (2009).

10. Senft, D. \& Ronai, Z. A. UPR, autophagy, and mitochondria crosstalk underlies the ER stress response. Trends Biochem. Sci. 40, 141-148 (2015).

11. Pehar, M., Jonas, M. C., Hare, T. M. \& Puglielli, L. SLC33A1/AT-1 protein regulates the induction of autophagy downstream of IRE1/XBP1 pathway. J. Biol. Chem. 287, 29921-29930 (2012).
12. Hernandez, L. D., Pypaert, M., Flavell, R. A. \& Galan, J. E. A. Salmonella protein causes macrophage cell death by inducing autophagy. J. Cell Biol. 163, 1123-1131 (2003).

13. Xue, Y. et al. Host inflammatory response inhibits Escherichia coli O157:H7 adhesion to gut epithelium through augmentation of mucin expression. Infect. Immun. 82, 1921-1930 (2014). 